\title{
Diversidad, historia natural y conservación de los mamíferos de San Vito de Coto Brus, Costa Rica
}

\author{
Jesús Pachecoํㅡ, Gerardo Ceballos ${ }^{1}$, Gretchen C. Daily², Paul R. Ehrlich², Gerardo Suzán ${ }^{1}$, \\ Bernal Rodríguez-Herrera ${ }^{1,3}$ \& Erika Marcé $^{1}$ \\ 1 Instituto de Ecología, Universidad Autónoma de México, Apartado Postal 70-275, México D.F. 04510, México. Fax: \\ 52-55-5622-9004; gceballo@miranda.ecologia.unam.mx. \\ 2 Center for Conservation Biology, Dept. Biological Sciences, Stanford University, Stanford, CA 94305-5020, USA. \\ Fax: 650- 723-3171. \\ 3 Historia Natural, Museo Nacional de Costa Rica, 749-1000 San José, Costa Rica, bernalr@racsa.co.cr; Fax (506) 233 \\ 7164.
}

Recibido 16-I-2003. Corregido 20-II-2004. Aceptado 12-III-2004.

\begin{abstract}
Diversity, natural history and conservation of mammals from San Vito de Coto Brus, Costa Rica. Although Costa Rica has been biologically well studied, few areas have complete mammal inventories, which are essential for ecological studies and conservation. The San Vito region is considered among the most important for scientific research in the country because of the presence of the Wilson Botanical Garden and Las Cruces. However, the knowledge of its mammalian fauna is incomplete. We extensively studied the mammals of San Vito, compiled a checklist, and evaluated its composition, relative abundance, habitat distribution, and conservation status. We recorded 105 species, representing 85 genera, 29 families, and 10 orders. Non-volant mammals represented 62 species, 59 genera, 23 families, and 9 orders. Bats belonged to 6 families, 26 genera and 43 species. The extensive deforestation and hunting have caused the extinction of seven species, but the region still supports, surprisingly, a relatively high number of species, most of which are rare. Few species are common and abundant. Species richness was higher in forest, and forest fragments; fewer species were found in coffee plantations, induced grasslands, and secondary vegetation. Around 21\% (13 species) are included in the IUCN red book. Three species are considered endangered (Saimiri oerstedii, Tapirus bairdii, and Sylvilagus dicei), and two threatened (Myrmecophaga trydactila and Caluromys derbianus), of which two (T. bairdii and M. trydactila) are locally extinct. The other species in IUCN are either of low risk (i.e. Chironectes minimus) or data deficient (Lontra longicaudis). Additionally, 24 species (39\%) are included in CITES. Rev. Biol. Trop. 54(1): 219-240. Epub 2006 Mar 31.
\end{abstract}

Key words: Mammals, bats, inventories, San Vito, Costa Rica, distribution, biogeography, conservation.

Los inventarios actualizados de especies son esenciales entre otras cosas, para llevar a cabo estudios ecológicos, de conservación y manejo (e.g. WCMC 1992, Wilson 1993). A pesar de que Costa Rica es un país que cuenta con estudios mastozoológicos desde mediados del siglo XIX (Rodríguez-H y Wilson 1999), pocas localidades incluyendo las áreas protegidas cuentan con inventarios de especies. Las localidades con mayor información han sido el bosque seco de Santa Rosa (Guanacaste) y el húmedo de Finca La Selva (Puerto Viejo de
Sarapiquí), ambas localidades de tierras bajas (Janzen 1983). Las tierras medias y altas de Costa Rica han sido poco estudiadas y la región menos conocida es la Cordillera de Talamanca con muy pocos registros en esa zona de estudio (Rodríguez-H. et al. 2002).

La región de San Vito de Coto Brus en el sur de Costa Rica es considerada como una de las más importantes del país para la investigación científica, la cual ha sido promovida principalmente por el Jardín Botánico Wilson (JBW) y la Estación de Biología La Cruces (EBLC) de 
la Organización de Estudios Tropicales, establecidas a finales de la década de 1960 (Janzen 1983). Sin embargo, no existe un inventario de especies actualizado de la región, aunque hay registros desde principios del Siglo XX. En 1983, Don E. Wilson compiló la primera lista de mamíferos de San Vito y registró a 47 especies (Wilson 1983). Posteriormente, Rodríguez y Chinchilla (1996) reportaron 53 especies en la región. En ambos trabajos, sin embargo, se registraron a las especies presentes en tiempos históricos, sin evaluar su estado actual de conservación.

La región de San Vito se ha transformado notablemente debido a la intensa deforestación que ha ocurrido desde hace 40 años provocando la pérdida de la mayor parte de su vegetación natural. Hay evidencias sólidas de que la deforestación y el cambio en el uso del suelo, han causado impactos negativos severos en la fauna y flora (Ceballos et al. 1999, Daily et al. 2001, Hughes et al. 2002), como la extinción local de especies de algunos grupos, cambios en la abundancia de las especies sobrevivientes, y la invasión de especies nativas y exóticas (Daily et al. 2003). Por lo tanto, el objetivo de este trabajo es el de evaluar la composición, diversidad y situación actual de los mamíferos de San Vito. Este estudio es complementario al de Daily et al. (2003), que se enfocó a evaluar específicamente al efecto de la fragmentación del hábitat en la diversidad de especies de las comunidades de mamíferos.

\section{MATERIAL Y MÉTODOS}

El área de estudio comprende un radio de aproximadamente $20 \mathrm{~km}$ de la Estación Biológica de Campo Las Cruces (EBC) de la Organización de Estudios Tropicales (OTS). La Estación se encuentra a $1100 \mathrm{msnm}$, y se localiza a $5 \mathrm{~km}$ al sur de San Vito de Coto Brus, Provincia de Puntarenas, y a unos $30 \mathrm{~km}$ de la

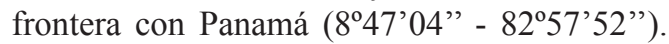
Parte de la transformación que ha sufrido la región de San Vito tiene como consecuencia la pérdida y fragmentación de su cubierta forestal.
Las áreas deforestadas han sido convertidas en pastizales, cultivos de café y huertos con mezclas de especies como café, yuca, piña, papaya, plátano y mango. Actualmente, solo el $25 \%$ del área permanece forestada, en fragmentos generalmente menores de 30 ha. El fragmento de mayor tamaño es la EBC, que tiene aproximadamente 200 ha, y es la única zona protegida de la región (Daily et al. 2001, 2003). Aproximadamente $20 \%$ de las áreas deforestadas son utilizadas para cultivos de café, $30 \%$ para pastizales y el resto para cultivos de yuca, plátano, $\mathrm{y}$ otros vegetales.

La zona de vida es el bosque lluvioso tropical premontano según la clasificación de Holdridge (1967), que se caracteriza por presentar dos o tres estratos en donde los árboles del dosel presentan una altura de 30 a $40 \mathrm{~m}$ y los del sotobosque de 10 a $20 \mathrm{~m}$. La cobertura del dosel es variable y entre los árboles dominantes están varias especies de encinos (Quercus spp). Tiene abundante epifitas que incluyen bromélias, orquídeas, musgos y líquenes. El sotobosque es relativamente denso y su composición de especies es diversa (Hartshorn 1983). La topografía del paisaje es montañosa con una elevación entre 1120 y $1385 \mathrm{msnm}$, con un intervalo desde 400 hasta $1800 \mathrm{msnm}$. La temperatura y la precipitación anual promedio son de $22^{\circ} \mathrm{C}$ y $3400 \mathrm{~mm}$, respectivamente, con una estación seca con poca lluvia de enero a abril.

El trabajo de campo se llevó a cabo principalmente entre febrero y mayo de 1999, con periodos adicionales en marzo del 2001 y marzo del 2002. En el 2001 y 2002 el trabajo de campo consistió básicamente en observaciones directas, que se concentraron en la búsqueda de especies en remanentes de bosque. En 1999 se realizaron muestreos en 27 localidades dispersas en la región de San Vito de Coto Brus. Las localidades se seleccionaron para representar un bosque continuo (JBW), fragmentos de bosque asociados a cafetales o pastizales, cafetales y pastizales. Todas las localidades se revisaron 3 veces durante 2 días consecutivos. Para la captura de mamíferos pequeños se delimitaron cuadrantes de 0.49 ha, con 64 trampas Sherman en un arreglo de 8 filas y 8 columnas, con una 
separación entre trampas de $10 \mathrm{~m}$ y cebadas con una mezcla de avena, crema de cacahuate y vainilla. El número total de trampas-noche fue de 7938.

Los mamíferos medianos y grandes se registraron por medio de observaciones directas, trampas Tomahawk y trampas de arena. Las trampas Tomahawk se colocaron en cuadrantes de 0.8 ha, en un arreglo de 3 X 3, con una separación de $30 \mathrm{~m}$ entre cada trampa. Se cebaron con sardina y pedazos de plátano. El mismo arreglo y número de trampas se utilizó para las trampas de arena, que se cebaron con sardina y plátano. El número de noches- trampa en ambos casos fue de 1458 .

Los mamíferos voladores no se estudiaron con relación a la fragmentación, por lo que solo se presentan los resultados de riqueza y la lista de las especies de acuerdo a la revisión bibliográfica de Wilson (1983), Rodríguez y Chinchilla (1996), Rodríguez-H y Wilson (1999) y LaVal y Rodríguez-H (2002), y también por ejemplares depositados en American Museum of Natural History (AMNH), British Museum, Londres (BM), Field Museum of Natural History, Chicago (FMNH), Museum of Natural History, University of Kansas, Lawrence (KU), Natural History Museum of Los Angeles County, Los Angeles (LACM), Lousiana State University Museum of Zoology, Lousiana State University (LSUMZ), Museo Nacional de Costa Rica, San José (MNCR), Texas Cooperative Wildlife Collection, Texas A\&M, College Station, United States National Museum of Natural History (NMNH). Además se utilizaron los datos de grabaciones esporádicas del año 2001 y 2002, hechas con el Sistema Anabat II, por Bernal Rodríguez-Herrera.

Todos los mamíferos capturados fueron marcados con un arete en la oreja derecha, medidos y liberados. La identificación de las especies se llevó a cabo con la ayuda de las guías de campo de Ceballos y Miranda (1986, 2000) y Reid (1997). Para la identificación de huellas se siguió a Aranda (2001).

Con relación a sus afinidades de hábitat los mamíferos se clasificaron como especialistas de bosque (i.e. las especies exclusivas de ese hábitat), generalistas de bosque (i.e. aquellas especies que dependen del bosque pero pueden utilizar ambientes perturbados), generalistas (i.e. especies que sobreviven en ambientes naturales y perturbados) e invasoras (i.e. aquellas especies que han invadido la región al incrementarse los ambientes perturbados) de acuerdo a la información reportada en Ceballos y Miranda (1986, 2000) y Reid (1997).

Las especies se clasificaron de acuerdo a $\mathrm{su}$ abundancia relativa regional como localmente extintas, raras, comunes y abundantes. Las especies raras tuvieron menos de 6 registros; las comunes de 7 a 10 registros y las abundantes 11 ó más registros. La estimación de la abundancia relativa debe tomarse como una aproximación, ya que existen diversos factores, como el tipo de muestreo y los hábitats que se estudiaron, que no pueden controlarse correctamente para hacer comparaciones finas entre los diferentes grupos de especies, como las arborícolas y las terrestres. Sin embargo, debido a que fueron sistemáticos proporcionan una base cualitativa sólida para comparar tendencias entre esos grupos.

\section{RESULTADOS}

En total se compilaron registros de 105 especies, que representaron a 85 géneros, 29 familias y 10 órdenes (Fig. 1, Apéndices 1 y 2). Los mamíferos no voladores estuvieron representados por 62 especies, 59 géneros, 23 familias y 9 órdenes (Apéndice 2). Los mamíferos voladores (Orden Chiroptera) pertenecieron a 6 familias, 26 géneros y 43 especies (Apéndice 1).

El éxito de trampeo para mamíferos no voladores fue del $5 \%$, con un mayor número de registros en las trampas de arena (10.1\%), seguida de las Tomahawk (3.4\%) y las Sherman (1.7\%). De los mamíferos no voladores 23 especies solo se registraron a través de registros en la literatura y/o de informantes locales. Se hicieron registros directos de 17 especies (28\%), como la ardilla pigmea (Microsciurus alfari) y el oso hormiguero 


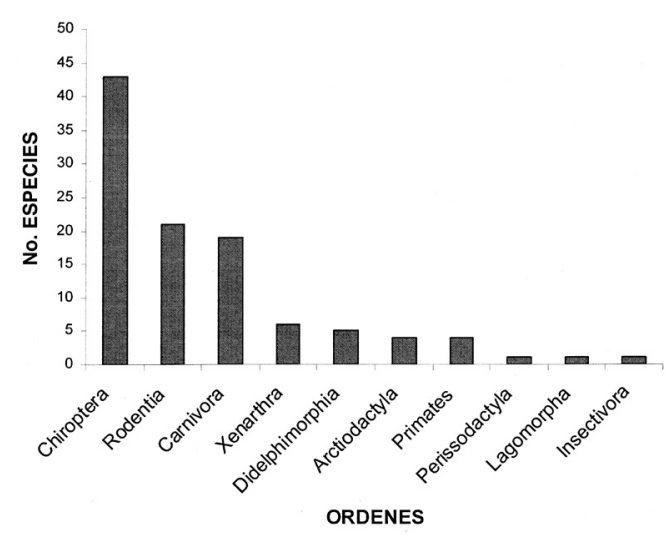

Fig. 1. Riqueza de especies de los órdenes de mamíferos de la región de San Vito de Coto Brus, Costa Rica.

(Tamandua mexicana). Hubo registros directos e indirectos (huellas) de 13 especies (21\%), como el zorro pelón (Didelphis marsupialis) y la nutria o perro de agua (Lontra longicaudis). Finalmente, se registraron indirectamente a 9 especies (15\%) como la comadreja (Mustela frenata) y el manigordo (Leopardus pardalis). De las 43 especies de murciélagos, 33 especies se registraron con ejemplares depositados en las colecciones consultadas, 5 por grabación ultrasónica y 5 por revisión de literatura.

Composición y riqueza de especies: El $41 \%$ de las especies pertenecieron al orden Chiroptera, lo que representa 8 especies más que las reportadas por Wilson (1983) y 7 más que Rodríguez y Chinchilla (1996). Se obtuvieron los primeros registros en la región de Centronycteris centralis, Pteropteryx macrotis, Saccopteryx leptura, Micronycteris microtis, Carollia castanea, Eptesicus furinalis, Lasiurus blossevillii y Molossus ater. Las especies identificadas por grabación (5) aún no cuentan con ejemplares colectados; sin embargo, la distribución probable reportada por LaVal y RodriguézH (2002) incluye a nuestra zona de estudio.

De las 62 especies de mamíferos no voladores registrados, $66 \%$ pertenecen a los órdenes Rodentia y Carnivora (Apéndice 2); el resto de los órdenes estuvo representado por menos de 10 especies. No se encontraron especies endémicas del país, y en general la mayoría (90\%) presentaron distribuciones amplias; sólo seis especies (Cryptotis nigrescens, Saimiri oerstedii, Orthogeomys cavator, Rheomys raptor, Leopardus tigrinus y Sylvilagus dicei) fueron de distribución restringida (Apéndice 2).

Distribución por tipo de vegetación: Como era de esperarse, la mayoría de las especies $(55 \%)$ se registraron en el bosque de la $\mathrm{EBC}$, seguido por los fragmentos de bosques, fragmentos - cafetal, fragmentos - pastizal, cafetal y pastizal (Fig. 2). El mayor número de especies exclusivas a un ambiente (11 especies, $25 \%$ ) se encontró en el bosque continuo y en los fragmentos de bosques. Sólo se registró a una especie como exclusiva del pastizal (la rata Sigmodon hispidus) y otra en cafetales asociados a fragmentos de bosque (el zorro Urocyon cinereoargenteus).

Abundancia relativa: La mayoría de las especies (54\%), tales como el puma (Puma concolor), grisón (Galictis vittata) y cabro de monte (Mazama americana), fueron raras de acuerdo a la clasificación de abundancia usada (Fig. 2, Apéndice 2). Las especies más comunes y abundantes en el bosque continuo y los fragmentos de bosque fueron el zorro pelón (Didelphis

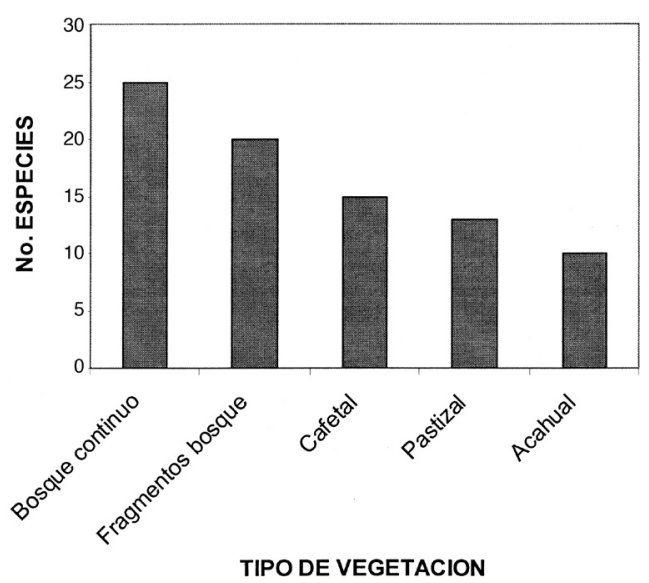

Fig. 2. Riqueza de especies en los principales tipos de vegetación natural y modificada por actividades antropogénicas de la región de San Vito de Coto Brus, Costa Rica. 
marsupialis), el cariblanco (Cebus capucinus) y la ardilla roja (Sciurus granatensis); en los pastizales y cafetales las más comunes fueron los zorros (D. marsupialis, Philander oppossum, Marmosa mexicana) y una rata (Olygorizomys fulvescens). Entre los roedores, los más comunes fueron las ratas Melanomys caliginosus y Oligoryzomys fulvescens.

Estado de conservación: Por lo menos el $11 \%$ de las especies registradas en la región, incluyendo al jaguar (Panthera onca) y el mono congo (Alouatta palliata), están localmente extintas (Apéndice 2). Sin embargo, a pesar de la extensa deforestación y fragmentación del bosque original, se encontró una alta persistencia de especies.

El 21\% (13) de las especies se encuentran en la lista roja de la Unión Internacional para la Conservación de la Naturaleza (IUCN) (HiltonTaylor 2000). De estas el mono ardilla (Saimiri oerstedii), el danta (Tapirus bairdii) y el conejo (Sylvilagus dicei) son consideradas en peligro de extinción (EN); el oso caballo (Myrmecophaga tridactila) y el zorro (Caluromys derbianus) son vulnerables (V); el zorro de agua (Chironectes minimus), el olingo (Bassaricyon gabbii) y el cacomixtle (Bassariscus sumichrasti) se consideran como de bajo riesgo (LR/nt). Finalmente, la perica ligera (Choloepus hoffmanni), el perro de agua (Lontra longicaudis) y el cabro de monte (Mazama americana) se incluyen con datos insuficientes (DD) y el jaguar (Panthera onca) y el tigrillo (Leopardus tigrinus.) casi amenazadas (NT). Es interesante notar que la situación actual de varias de estas especies en la región es diferente a la consignada por la UICN. Un claro ejemplo son el oso caballo y el jaguar, extirpados localmente y en riesgo globalmente.

Adicionalmente, 24 especies (39\%) se encuentran en algún apéndice de la Convención Internacional sobre Tráfico de Especies en Peligro de Flora y Fauna (CITES, 2001). Pertenecen al Apéndice I monos ( $S$. oerstedii, $A$. palliata) y carnívoros (L. longicaudis, $P$. onca, P. concolor, L. pardalis, L. tigrinus, L. weidii y $H$. yaguarondi). En el Apéndice II se encuentran los xenarthros (M. tridactila y B. variegatus).
Finalmente, en el Apéndice III se encuentran un xenarthro y tres carnivoros (C. hoffmanni, $G$. vittata, B. gabbii y B. sumichrasti).

\section{DISCUSIÓN}

El éxito de muestreo de este trabajo fue mayor a los obtenidos comúnmente en el Neotrópico, donde las densidades y los esfuerzos de muestreo son muy bajos especialmente en roedores (e.g. Ceballos y Miranda 2000, Voss y Emmons 1996). Por ejemplo, el éxito de muestreo en un estudio en el Amazonas, utilizando más de 100000 noches-trampa fue menor del 1\% (Hice y Schmidly 2002).

El éxito de muestreo obtenido en las trampas de arena fue alto, a pesar de sus limitaciones, este método aporta información adicional a otros tipos de muestreo más tradicionales. Sin embargo, este método es poco empleado en proyectos de inventarios, ecológicos y de conservación. Otro punto a resaltar es que a pesar del notable esfuerzo de muestreo con trampas Sherman no capturamos musarañas; sin embargo, capturamos varios individuos en trampas de caída ("pitfall traps"), que eran baldes de plástico de 20 lts enterrados, en un estudio sobre los reptiles y anfibios de la región. Esto es similar a lo que ocurre con musarañas en otros ambientes templados y tropicales (ver Hice y Schmidly 2002). Por ejemplo, la musaraña Megasorex gigas no se colectó en más de 100000 nochestrampa en un bosque tropical caducifolio en la reserva de la biosfera Chamela - Cuixmala, en Jalisco, México, y sólo fue registrada cuando se usaron trampas de caída (Téllez et al. 1997, Ceballos y Miranda 2000).

Los resultados obtenidos para los murciélagos son producto de una revisión, y por supuesto con trabajo sistemático que involucre un mayor esfuerzo de captura con redes, búsqueda de refugios, la lista de especies aumentaría. En especial al trabajo con ténicas de grabación es prometedor ya que por éstas se han encontrado nuevos registros en lugares inesperados y en elevaciones superiores a las previstas (LaVal y Rodríguez-H 2002). 
Choeroniscus godmani fue registrada por Rodríguez y Chinchilla (1996), sin embargo, en trabajos posteriores no se encontró en la región (Rodríguez-H y Wilson 1999, Timm et al. 1999). Choeronycteris mexicana registrada por Rodríguez y Chinchilla (1996) aún no cuenta con ejemplares de Costa Rica (Rodríguez-H y Wilson 1999) y su distribución actual es de Estados Unidos hasta Honduras (Reid 1997). El registro de Chiroderma salvini se basa en un ejemplar de LACM, sin embargo esta especie no esta registrada para el pacífico sur de Costa Rica (Rodríguez-H y Wilson 1999, LaVal y Rodríguez-H 2002).

La composición de la mastofauna de San Vito es dinámica, ya que ha sufrido extinciones, variaciones en las abundancias, y adiciones de especies. Por lo menos 7 especies (e.g. el mono araña $A$. geoffroyi) se consideran localmente extintas. Es posible que otras especies que están registradas en la literatura se encuentren en la región, pero no se pudo registrar en nuestro trabajo de campo, como Hoplomys gymnurus, Proechimys semispinosus, Orthogeomys cavator y Rheomys raptor, por que se encuentran en ambientes y microhábitats que no se incluyeron en el muestreo.

Dadas las características de los ambientes que se muestrearon, esperabamos encontrar a Heteromys desmarestianus, Scotinomys teguina, Peromyscus mexicanus y Reithrodontomys mexicanus, sin embargo, no capturamos ninguna de estas especies, posiblemente porque sus densidades son muy bajas debido a las condiciones de fragmentación actuales, lo que explicaría su ausencia.

Otras dos especies, Chironectes minimus y Scotinomys teguina que son muy susceptibles a las perturbaciones del hábitat, probablemente representan extinciones locales adicionales, sin embargo, la información que tenemos no nos permite precisar su situación actual. Por último dos especies nativas invasoras Canis latrans y Oryzomys couesi, todavía son localmente raras.

Por el contrario, el registro del mono tití (Saimiri oerstedii) en la zona, representa una importante extensión del área de distribución de esta especie considerada en peligro de extinción (Ceballos, obs. pers., Hilton-Taylor 2000). Otros registros interesantes son los de Cyclopes didactylus, Microsciurus alfari, Tylomys watsoni, Lontra longicaudis, Galictis vittata, Puma concolor y Leopardus tigrinus, especies con pocos registros anteriores en la región o en Costa Rica, algunas de las cuales se encuentran en peligro de extinción.

Es importante resaltar la alta contribución de los fragmentos de cafetal a la riqueza regional de especies. Esto se debe principalmente a que los cafetales se encuentran en contacto con fragmentos de bosque, lo que ofrece además de refugio a la fauna silvestre, alimento y lugares con cobertura vegetal adecuados para que se desplacen. Las especies comunes y abundantes fueron poco sensibles a las perturbaciones antrópicas, e inclusive se favorecieron con la conversión del bosque en pastizales y cultivos, ejemplos de estas especies son los roedores Zygodontomys brevicauda y Olygoryzomys fulvescens. Sin embargo, especies como el tolomuco (Eira barbara) y el mono carablanca (Cebus capucinus) aún son relativamente comunes, a pesar de la modificación que ha sufrido la vegetación natural.

Nuestras categorías de abundancia, reflejan la situación regional de las especies; sin embargo, cuando se consideran regiones más amplias esta clasificación coincide en algunas especies, como el del puma y la guatusa (Dasyprocta punctata), y para otras es muy diferente tal es el caso del mono colorado (Ateles geoffroyi), que en la region de San Vito esta extinto pero mantiene poblaciones numerosos en otras partes de Costa Rica.

\section{AGRADECIMIENTOS}

Agradecemos a Bryce Stephens, Jenna Mieren y Jesús Ilama por su colaboración en el trabajo de campo. Nuestros amigos y colegas del Jardín Botánico Wilson, especialmente Luis Diego Gómez, Gail Hewson y Rodolfo Quiroz, compartieron con nosotros información sobres sus observaciones de mamíferos en la región y 
apoyaron con la logística de este trabajo. El Sr. Jorge Pérez y la Familia Gamboa nos permitieron el acceso a sus fincas y diligentemente respondieron nuestras numerosas preguntas. Jorge Pérez nos acompaño en varias salidas al campo y nos proporcionó la información más detallada de los mamíferos de la región. Al Ing. Javier Guevara Sequeira del Ministerio del Ambiente y Energía (MINAE) de Costa Rica por otorgarnos los permisos de colecta para realizar la investigación. Nuestro colega tico, Federico Chinchilla, leyó críticamente una versión previa del manuscrito.

\section{RESUMEN}

Aunque Costa Rica ha sido biológicamente bien estudiada, pocas áreas tiene inventarios de mamíferos completos y actualizados, lo que es para llevar a cabo estudios ecológicos y de conservación. La región de San Vito es considerada entre las mas importantes para la investigación científica en el país, debido a la presencia del Jardín Botánico Wilson y la Estación Biológica Las Cruces. Sin embargo, el conocimiento de la mastofauna es incompleto. Por lo tanto se evaluó intensamente los mamíferos de San Vito, y se compiló una lista de especies, con una evaluación de su composición, abundancia relativa, distribución en los habitats, y su estado de conservación. Se registraron 105 especies, que representaron a 85 géneros, 29 familias y 10 órdenes. Los mamíferos no voladores representaron 62 especies, 59 géneros, 23 familias y 9 órdenes. Los murciélagos pertenecieron a 6 familias, 26 géneros y 43 especies. La extensa deforestación y cacería son factores que han causado la extinción local de 7 especies, pero la región todavía mantiene un número relativamente alto de su diversidad original, incluyendo a especie raras. Pocas especies fueron comunes o abundantes. La riqueza de especies fue alta en el bosque y los fragmentos de bosque, y más baja en las plantaciones de café, pastizales inducidos y vegetación secundaria. Alrededor del 21\% (13) de las espcies estan incluidad en el libro rojo de la UICN. Tres especies (Saimiri oerstedii, Tapirus bairdii y Sylvilagus dicei) son consideradas en peligro y dos (Myrmecophaga trydactila y Caluromys derbianus) como amenazadas: de estas, dos especies (T. bairdii and M. trydactila) se encuentran localmente extintas. Las otras especies son consideradas de bajo riesgo (i.e. Chironectes minimus) o con poca información (i.e. Lontra longicaudis). Adicionalmente, 24 especies (39\%) están incluidas en CITES.

Palabras clave: Mamíferos, murciélagos, inventario, San Vito, Costa Rica, distribución, biogeografía, conservación.

\section{REFERENCIAS}

Aranda, M. 2001. Huellas y otros rastros de los mamíferos grandes y medianos de México. CONABIO - Instituto de Ecología, México D.F.

Ceballos, G. \& A. Miranda. 1986. Los Mamíferos de Chamela, Jalisco: manual de campo. Instituto de Biología, UNAM, México. 299 p.

Ceballos, G., G. Daily, P. Ehrlich, J. Pacheco, G. Suzan, A. Sanchez-Ozofeifa, B. Stephens \& J. Mieren. 1999. Countryside Biogeography of Neotropical Mammals: A Costa Rican Case Study. Amigos Newsletter, Wilson Botanical Garden, Las Cruces Biological Field Station, Costa Rica. 52: 5-7.

Ceballos, G. \& A. Miranda. 2000. Guía de Campo de los Mamíferos de la Costa de Jalisco / A field guide to the mammals of the Jalisco coast. Fundación Ecológica de Cuixmala A.C., México. 424 p.

CITES. 2001. Annotated CITES Appendices and Reservations. UNEP-World Conservation Monitoring Center, Ginebra, Suiza.

Daily, G.C., P.R. Ehrlich \& A. Sánchez-Azofelia. 2001. Countryside biogeography: Use of human dominated habitats by the avifauna of southern Costa Rica. Ecol. Appl. 11: 1-13.

Daily, G.C., G., G. Ceballos, J. Pacheco, G. Suzán \& A. SánchezAzofeifa. 2003. Countryside Biogeography of Neotropical Mammals: Conservation Opportunities in Agricultural Landscape of Costa Rica. Conserv. Biol. 17: 1-11.

Gardner, A.L., R.K. LaVal \& D.E. Wilson. 1970. The distribution status of some Costa Rican bats. J. Mammal. 51: 712-729.

Hartshorn, G.S. 1983. Plants, p.118-157. In D.H. Janzen (ed.). Costa Rican Natural History. University Chicago, Chicago.

Hewson, G. 1996. Fauna of Las Cruces. Amigos Newsletter. 49: 3 .

Hice, Ch.L. \& D.J. Schmidly. 2002. The effectiveness of pitfall traps for sampling small mammals in the Amazon basin. Mastozool. Neotrop. 9: 85-89.

Hilton-Taylor, C. 2000. 2000 IUCN red list of threatened species. IUCN, Glanz, Suiza.

Holdridge, L.S. 1967. Life zone ecology. Trop. Sci. Center, San José, Costa Rica.

Hughes, J.B., G.C. Daily \& P.R. Ehrlich. 2002. Agricultural policy can help reserve tropical forest birds in countryside habitats. Ecol. Letters 5: 121-129. 
ICZN. 1998. Opinion 1894. Regnum Animale..., Ed. 2 (M.J. Brisson, 1762): rejected For nomenclatural purposes, with the conservation of the mammalian generic names for Philander (Marsupialia), Pteropus (Chiroptera), Glis, Cuniculus and Hydrochoerus (Rodentia), Meles, Lutra and Hyaena (Carnivora), Tapirus (Perissodactyla), Tragulus and Giraffa (Artiodactyla). Bull. Zool. Nom. 55: 64-71.

Janzen, D.H. 1983. Costa Rican Natural History. University of Chicago, Chicago.

LaVal R.K. \& B. Rodríguez-H. 2002. Murciélagos de Costa Rica. Instituto Nacional de Biodiversidad, San José, Costa Rica.

Reid, F.A. 1997. A field guide to the mammals of Central America and Southeast Mexico. Oxford University, Nueva York.

Rodríguez, J. \& F. Chinchilla. 1996. Lista de los mamíferos de Costa Rica. Rev. Biol. Trop. 44: 877-890.

Rodríguez-H., B. \& D.E. Wilson. 1999. Lista y distribución de las especies de murciélagos de Costa Rica. Occasional Papers, Conserv. Int. 5: 1-34.

Rodríguez-H., B., D.E. Wilson, M. Fernández \& W. Pineda. 2002. El estudio de la mastofauna de Costa Rica: Historia, recolecta, localidades y riqueza de especies. Manuscrito inédito.
Rodríguez-Herrera, B., F.A. Chinchilla \& L.L. May. 2002. Lista de especies, endemismo y conservación de los mamíferos de Costa Rica. Rev. Mex. Mastozool. 6: 19-41.

Téllez, G., A. Mendoza \& G. Ceballos. 1997. Registros notables de mamíferos del oeste de México. Rev. Mex. Mastozool. 2: 97-100.

Timm, R.M., R.K. LaVal \& B. Rodríguez-H. 2000. Clave de Murciélagos de Costa Rica. Brenesia 52: 1-32.

Voss, R. \& L.H. Emmons. 1996. Mammalian diversity in Neotropical lowland rainforests: a preliminary assessment. Bull. Am. Museum Nat. Hist. 230: 1-115.

WCMC (World Conservation Monitoring Centre). 1992. Global biodiversity. WCMC, Cambridge, Gran Bretaña.

Wilson, D.E. 1983. Checklist of Mammals. pp. 443-447. In D.H. Janzen (ed.). Costa Rican natural history. Chicago University, Chicago.

Wilson, E.O. 1993. The diversity of life. Norton, New York.

Woodman, N. \& R. M. Timm. 1993. Intraespecific and interespecific variation in the Cryptotis nigrescens species complex of small-eared shrews (Insectivora: Soricidae), with the description of a new species from Colombia. Fieldiana Zool. 74: 1-30. 


\section{APÉNDICE 1}

Lista sistemática de los murciélagos de la región de San Vito, Coto Brus, Costa Rica. Se indica la fuente de información que registra la especie para la región. Los ejemplares de museo son un testigo, no necesariamente el único existente. $\mathrm{KU}=$ Museum of Natural History, University of Kansas, Lawrence. LACM= Natural History Museum of Los Angeles County, Los Angeles MNCR= Museo Nacional de Costa Rica. TCWC = Texas Cooperative Wildlife Collection, Texas A\&M. UCR= Museo de Zoología Universidad de Costa Rica.

TAXA

FUENTE

\section{ORDEN CHIROPTERA}

Familia Emballonuridae

Centronycteris centralis . . . . . . . . . . . . Grabación (Rodríguez-H com pers)

Pteropteryx macrotis . . . . . . . . . . . . . . . . Grabación (Rodríguez-H com pers)

Saccopteryx bilineata . . . . . . . . . . . . . . . . (LaVal y Rodríguez-H 2002)

Saccopteryx leptura . . . . . . . . . . . . Grabación (Rodríguez-H com pers)

Familia Mormoopidae

Pteronotus parnellii. . . . . . . . . . . . . . . . . . . . . . . LACM-038669

Familia Phyllostomidae

Lonchorhina aurita . . . . . . . . . . . . . . . . . . . . . . . LACM-038674

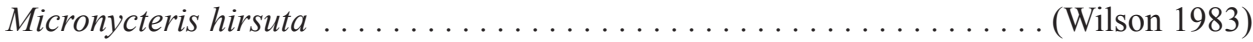

Micronycteris microtis. . . . . . . . . . . . . . . . . . . . LACM-038671

Phyllostomus discolor . . . . . . . . . . . . . . . . . . . . . LACM-038673

Phyllostomus hastatus . . . . . . . . . . . . . . . . . . . . . (Wilson 1983)

Lonchophylla mordax . . . . . . . . . . . . . . . . . . . . . . LACM-038676

Lonchophylla robusta . . . . . . . . . . . . . . . . . . . . . . LACM-038675

Anoura cultrata . . . . . . . . . . . . . . . . . . . . . . . LACM-038682

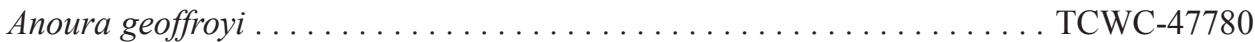

Glossophaga commissarisi . . . . . . . . . . . . . . . . . . . . . . LACM-038681

Glossophaga soricina . . . . . . . . . . . . . . . . . . . . . . . MNCR-1132

Hylonycteris underwoodi . . . . . . . . . . . . . . . . . . (Gardner et al. 1970)

Carollia brevicauda . . . . . . . . . . . . . . . . . . . . . . . . . . LACM-038684

Carollia castanea . . . . . . . . . . . . . . . . . . . . . . . MNCR-1130

Carollia pespicillata . . . . . . . . . . . . . . . . . . . . . . LACM-042398

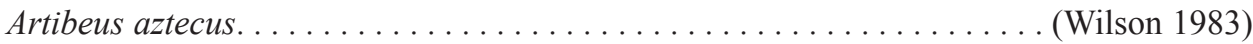

Artibeus jamaicensis . . . . . . . . . . . . . . . . . . . . . . . LACM-038689

Artibeus lituratus. . . . . . . . . . . . . . . . . . . . . . . MNCR-1131

Artibeus toltecus . . . . . . . . . . . . . . . . . . . . . . . . . . LACM-028746

Artibeus watsoni . . . . . . . . . . . . . . . . . . . . . . . . LACM-038693

Chiroderma salvini . . . . . . . . . . . . . . . . . . . . . . . . . LACM-038708

Chiroderma villosum . . . . . . . . . . . . . . . . . . . . . . LACM-038711

Enchisthenes hartii . . . . . . . . . . . . . . . . . . . . . . LACM-028750

Platyrrhinus helleri. . . . . . . . . . . . . . . . . . . . . . . . . LACM-028738

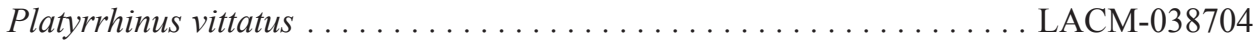

Sturnira lilium . . . . . . . . . . . . . . . . . . . . . . . . . . . LACM-028756

Sturnira ludovici . . . . . . . . . . . . . . . . . . . . . . . . . . . . LACM-038717 
Sturnira mordax. MNCR-1129

Uroderma bilobatum KU-117540

Vampyressa pusilla . . LACM-028752

Desmodus rotundus. LACM-038720

Familia Thyropteridae

Thyroptera tricolor

. UCR-461

Familia Vespertilionidae.

Eptesicus brasiliensis TCWC-47827

Eptesicus furinalis. .

TCWC-47828

Lasiurus blossevillii.

TCWC-47832

Myotis nigricans

Grabación (Rodríguez-H com pers)

Familia Molossidae.

Eumops auripendulus LACM-028774

Molossus ater Grabación (Rodríguez-H com pers) 


\section{APÉNDICE 2}

Lista sistemática de los mamíferos terrestres de la región de San Vito, Coto Brus, Costa Rica. Se indican su abundancia relativa y los ambientes en los que se registraron a las especies. También se indican las especies extintas y las no registradas en este estudio pero que tienen registros previos en la región, pero que se desconoce su situación actual.

TAXA

\section{ORDEN DIDELPHIMORPHIA}

Familia Marmosidae Marmosa mexicana

Familia Caluromydae Caluromys derbianus

Familia Didelphidae Chironectes minimus Didelphis marsupialis Philander opossum

\section{ORDEN XENARTHRA}

Familia Myrmecophagidae

Cyclopes didactylus

Myrmecophaga tridactyla Tamandua mexicana

Familia Megalonychidae Choloepus hoffmanni

Familia Bradypodidae Bradypus variegatus

Familia Dasypodidae Dasypus novemcinctus

\section{ORDEN INSECTIVORA}

Familia Soricidae Cryptotis nigriscens

\section{ORDEN PRIMATES}

Familia Cebidae

Saimiri oerstedii

Cebus capucinus

Alouatta palliata

Ateles geoffroyi

\section{ORDEN CARNIVORA}

Familia Canidae

Canis latrans

Urocyon cinereoargenteus
Abundancia

Común

Rara

Extinta

Abundante

Común

Rara

Extinta

Rara

Rara

No registrada

Común

Común

$\begin{array}{lllll}X & X & X & X & X\end{array}$

Rara

Común

Extinta

Extinta
Tipo de vegetación

BO FR CA PA OT


Familia Mephitidae

Conepatus semistriatus

Rara

Spilogale putorius

Rara

$\begin{array}{ccccc}X & X & X & X & X \\ X ? & X & X & X & ?\end{array}$

Familia Mustelidae

Eira barbara

Galictis vittata

Lontra longicaudis

Común

Rara

Rara

Mustela frenata

Común

$\begin{array}{ccccc}\text { X } & \text { X } & \text { X } & \text { X } & \text { X } \\ & \text { X } & \text { X? } & & \\ \text { X } & \text { X } & & & \text { X } \\ \text { X } & \text { X } & \text { X } & & \text { X }\end{array}$

Familia Procyonidae

Bassariscus sumichrasti

Rara

Bassaricyon gabbii

Rara

Nasua narica

Potos flavus

Común

Común

Procyon lotor

Común

Familia Felidae

Herpailurus yaguarondi

Rara

Leopardus pardalis

Rara

Leopardus tigrinus

Leopardus wiedii

Panthera onca

Rara

Rara

Extinta

Puma concolor

Rara

$\begin{array}{ccccc} & \text { X } & & & \\ & \text { X } & \text { X } & & \\ \text { X } & \text { X } & \text { X } & \text { X } & \text { X } \\ \text { X } & \text { X } & & \text { X } & \\ \text { X } & \text { X } & \text { X } & & \text { X }\end{array}$

X X X

X $\quad X \quad X$

$\mathrm{X}$

$\mathrm{X}$

X

X

ORDEN ARTIODACTYLA

Familia Tayassuidae

Tayassu pecari

Tayassu tajacu

Familia Cervidae

Mazama americana

Odocoileus virginianus

Extinta

Rara

Rara

Rara

Abundante

Rara

No registrada

No registrada

Microsciurus alfari

Familia Geomyidae

Orthogeomys cavator

Familia Heteromyidae

Heteromys desmarestianus

Familia Muridae

Melanomys caliginosus

Nyctomys sumichrasti

Oryzomys alfaroi

Oryzomys couesi

Oligoryzomys fulvescens
Abundante

Rara

Abundante

No registrada

Abundante
$\begin{array}{lll}X & X & X \\ & X & X\end{array}$

$\mathrm{X}$ 
Peromyscus mexicanus No registrada

Reithrodontomys mexicanus No registrada

Rheomys raptor

No registrada

Scotinomys teguina

Sigmodon hispidus

No registrada

Tylomys watsoni

Común

Rara

Zygodontomys brevicauda

Familia Erethizontidae

Coendou mexicanus

Familia Cuniculidae

Cuniculus paca

Familia Dasyproctidae

Dasyprocta punctata

Familia Echimydae

Hoplomys gymnurus

Abundante

$\begin{array}{llll} & & \mathrm{X} & ? \\ \mathrm{X} & \mathrm{X} & & \\ \mathrm{X} & \mathrm{X} & \mathrm{X} & ?\end{array}$

Rara

$\mathrm{X}$

Común

X $\quad X \quad X$

Común

X $\quad X$

Proechimys semispinosus

No registrada

No registrada

\section{ORDEN LAGOMORPHA}

Familia Leporidae

Sylvilagus dicei

Común

62
$\begin{array}{lllll}X & X & X & X & X\end{array}$

$\begin{array}{lllll}40 & 38 & 20 & 16 & 19\end{array}$

- $-38-20-16$

TOTAL 


\section{APÉNDICE 3}

Lista de especies de mamíferos no voladores de la región de San Vito, con observaciones sobre su distribución e historia natural. Las localidades donde se registraron ejemplares se identifican por un número (Ver Apéndice 4 para la información detallada de cada localidad).

\section{ORDEN DIDELPHIMORPHIA Familia Marmosidae}

Marmosa mexicana zeledoni Goldman 1917: El ratón tlacuache Zorricí fue una especie común que se registró en el bosque premontando de la EBC y fragmentos de bosque, acahuales, cafetales y pastizales. Se encontró una hembra con 7 crías en marzo del 2002. Localidades: 1,3, 6, 9, 11,14, 17, 18,19, 20,22, 23, 25, 26. Ejemplares: 27. Hay registros previos en la región en Agua Buena (Hall 1981).

\section{Familia Caluromydae}

Caluromys derbianus pallidus (Thomas 1899): El zorro de balsa fue una especie aparentemente rara, que sólo se registró en el bosque premontano de la $\mathrm{EBC}$, un fragmento de bosque en el Río Bajo Coto y en un charral en la carretera San Vito - Cd. Neilly. Es posible que la estimación de su abundancia este subestimada por la falta de muestreo en árboles. Localidades: 3, 31. Registros: 2. La especie ya había sido registrada en la región (Rodríguez y Chinchilla 1996).

\section{Familia Didelphidae}

Chironectes minimus panamensis Goldman 1914: Se tuvo informes del zorro de agua en el Río Java, cerca de la EBC y el Río Negro, un pequeño afluente del Coto Brus. Ambos informantes indicaron que hace más de una década no se han observado (Jorge Pérez y Norman Gamboa, com. pers.). Es posible que estén localmente extintos en la región de San Vito. Sin embargo, es posible que haya una población remanente en la confluencia de los ríos Coto Brus y Térraba.

Didelphis marsupialis caucae J.A. Allen 1900: El zorro pelón fue la especie más abundante en la región. Se encontró en el bosque premontano de la EBC, en fragmentos de bosque, cafetal y pastizal. El mayor número de registros se obtuvo en cafetal-bosque y cafetales. Localidades: 1, 3, 5, 6, 9, 12, 17, 18, 19, 20, 21, 23, 27, 40. Ejemplares: 31. La especie ya había sido registrada en la región (MNCR-432, Rodríguez y Chinchilla 1996, Wilson 1983).

Philander opossum fuscogriseus J.A. Allen 1900: El zorro cuatro ojos fue una especie abundante en el bosque premontano de la $\mathrm{EBC}$, fragmentos de bosque, cafetales y pastizales. Se tuvo el mayor número de registros en ambientes perturbados como cafetal - bosque. Localidades: 1, 3, 5, 6, 9, 12, 17, 18, 19, 20, 21, 23, 27, 40. Ejemplares: 31. La especie ya había sido registrada en la región (Rodríguez y Chinchilla 1996, Wilson 1983).

\section{ORDEN INSECTIVORA Familia Soricidae}

Cryptotis nigrescens (J.A. Allen 1895): A pesar de lo intenso del muestreo con trampas Sherman, varias musarañas sólo fueron colectadas en trampas de caída ("pitfall traps") en el bosque maduro y en pastizales. Las medidas de los ejemplares preservados en alcohol son las siguientes: Longitud total 80, 80; cola vertebral 20, 21; pata trasera: 10, 12; oreja desde la escotadura: 0. Localidades: 4. Ejemplares: 2. Hay 
registros previos de San Vito (LACM- 074351, Woodman y Timm 1993).

\section{ORDEN XENARTHRA Familia Myrmecophagidae}

Tamandua mexicana chiriquiensis J.A. Allen 1904: El tamandua es aparentemente común en la región, a pesar de que sólo se registró una vez en un acahual. La familia Gamboa y el guardabosques de la EBC indicaron que los ven frecuentemente. Localidades: 39. Ejemplares: 1.

Cyclopes dydactylus dorsalis (Gray 1865): Se encontraron dos ejemplares, un macho y una hembra, de balsita o ceibita, como se les llama localmente, en un árbol de guayaba en Fila de Cal. El árbol estaba a cinco metros del árbol más cercano, y a más de 100 metros del bosque más cercano. Las ceibitas se encontraban casi en la punta del árbol durmiendo. Al día siguiente los encontramos en el mismo árbol y dos días después en un árbol a más de 20 metros, al cual sólo pudieron llegar caminando. Localidades: 30. Ejemplares: 2.

\section{Familia Choloepidae}

Choloepus hoffmani hoffmani Peters 1858: El perezoso de dos dedos es una especie bien conocida por los habitantes locales, pero de acuerdo a los datos de este trabajo fue raro. Se registró en el bosque premontano de la EBC y en un fragmento de bosque. Se encontró en un encino (Quercus sp) y una Cecropia obtusifolia Localidades: 4. Ejemplares: 2. La especie ya había sido registrada en la región (MNCR-433, Rodríguez y Chinchilla 1996).

\section{Familia Bradypodidae}

Bradypus variegatus griseus (Graym 1871): El perezoso de tres dedos fue bien conocido para los informantes locales, quienes indicaron que es escaso en San Vito, pero más numeroso en las tierras bajas del Río Coto Brus, donde también registramos a Saimiri oerstedii.

\section{Familia Dasypodidae}

Dasypus novemcinctus fenestratus Peters, 1864: El cusuco o armadillo fue una especie común, aunque sólo se registró en ambientes perturbados, incluyendo fragmentos de bosque con pastizal y cafetal. Localmente se le caza de manera intensa, porque su carne es apreciada. Localidades: 4, 21, 23,4, 44. Ejemplares: 4. La especie ya había sido registrada en la región (Rodríguez y Chinchilla 1996).

\section{ORDEN PRIMATES Familia Cebidae}

Allouatta palliata aequatoralis Festa, 1903: El mono congo o aullador esta localmente extinto. Al parecer desapareció al igual que el mono colorado por la cacería indiscriminada, que fue facilitada por la fragmentación del hábitat. Los últimos registros son de principios de 1980. Las poblaciones más cercanas se localizan en el Cerro Paraguas, dentro de la Reserva Indígena Guaymí. La especie ya había sido registrada en la región (Rodríguez y Chinchilla 1996).

Ateles geoffroyi panamensis Kellogg and Goldman 1944: El mono colorado o araña esta localmente extinto. En 1944 fue registrado en Agua Buena y Coto (Hall 1981, Kellogg y Goldman 1944). Sin embargo, los informantes locales indicaron que los últimos ejemplares desaparecieron alrededor de 1980 por la cacería indiscriminada, que fue facilitada por la fragmentación del hábitat. Los últimos registros del Sr. Sergio Pérez (com. pers.) fueron en las selvas de la región donde se une los ríos Bajo Coto Brus y Térraba, en donde se registró al mono ardilla (S. oerstedii). Actualmente los monos araña persisten en los alrededores de la Estación Biológica de Las Alturas, en el Parque Internacional La Amistad. 
Cebus capucinus imitator Thomas 1903: El mono cariblanco es el único primate común en la región. En el bosque de la la EBC se registró una tropa de 23 individuos. Asimismo, se observó una tropa de por lo menos 15 monos en un fragmento de bosque en la Fila de Cal. Los rancheros locales informaron de su presencia temporal en varios de los fragmentos, donde se mueven en busca de alimento. Es simpatrico con Saimiri oerstedii. Localidades: 1, 2, 4, 30. Ejemplares: 44. La especie ya había sido registrada en la región (Rodríguez y Chinchilla 1996, Wilson 1983).

Saimiri oerstedii oerstedii (Reinhardt 1872): El mono tití es un nuevo registro para la región (Ceballos et al. en revisión). Fue raro el bosque premontano, arriba de $800 \mathrm{msnm}$, donde sólo se registraron 2 individuos en las márgenes del Río Java, dentro de la EBC. Al parecer estos individuos se desplazaron desde las partes bajas siguiendo el bosque ripario del Río Limón. Después de una búsqueda intensa se les ubicó en una extensa región a lo largo de los valles y barrancas de los ríos Coto Brus, Cotón y Limón. La vegetación natural ha sido intensamente perturbada, por lo que los monos utilizan un mosaico de fragmentos de bosque, acahuales, bosques riparios, huertos y cultivos. Se considera que dada las condiciones en las que se encuentra es una especie críticamente amenazada con la extinción. Localidades: 26, 32, 34, 35, 36, 37. Ejemplares: 58.

\section{ORDEN CARNIVORA Familia Canidae}

Canis latrans dickeyi Nelson 1932: El coyote es una especie que ha sido registrada en Costa Rica en las últimas décadas (Janzen 1983). En la región de San Vito se le empezó a observar en la década de 1980. Actualmente es aparentemente todavía raro (Jorge Pérez com pers). Norman Gamboa de San Vito lo ha visto ha visto en los pastizales aledaños a la EBC. La subespecie la hemos asignado a la más cercana mencionada por Hall (1981).
Urocyon cinereoargenteus costaricensis Goodwin 1938: La zorra gris fue una especie común en ambientes perturbados, que registramos en cafetales y cafetal-bosque. Es interesante que no se le encontró en el bosque de la EBC. Es una especie que incrementa sus densidades en sitios perturbados (Ceballos y Miranda 2000). Localidades: 19. Ejemplares: 1.

\section{Familia Felidae}

Herpailusus yagouarondi panamensis $\mathrm{J}$. A. Allen 1904: El león breñero fue registrado en ambientes perturbados, como fragmentos de bosque asociados a pastizales y cafetales. Los informantes locales indicaron que es el felino más común en la región. Localidades: 22, 25. Ejemplares: 4.

Leopardus pardalis mearnsi J.A. Allen 1902: Sorprendentemente el manigordo fue una especie que fue registrada en bosque de la EBC, y fragmentos de bosque asociados a pastizales y cafetales. Su registro en pastizales y cafetales siempre fue cerca de los fragmentos de bosque. Es posible que sobreviva en la región gracias al bosque continuo y su relación con fragmentos, especialmente los que se encuentran a lo largo de arroyos y ríos. Localidades: 1, 3, 11, 20, 21, 25. Ejemplares: 6 .

Leopardus tigrinus oncilla Thomas 1902: Este es aparentemente el primer registro de la especie en la región. El Sr. E. Laurent de San Vito, mostró una foto de un ejemplar que se mantuvo en cautiverio por varios meses y fue capturado como cría en una cañada cercana a San Vito. Localidades: 32. Ejemplares: 1.

Leopardus wiedii nicaraguae (J.A. Allen 1919). El causel fue una especie rara, que sólo se registró una vez en el bosque de la EBC. El guardaparques de la EBC nos indicó que es muy escaso. Localidades: 4. Registros: 1. registros adicionales: MNCR-431.

Panthera onca centralis (Mearns 1901): El jaguar o tigre es una especie regionalmente 
extinta, a pesar de que el guardabosques de la EBC indicó que observo un ejemplar juvenil en 1996. La familia Gamboa informó que el último ejemplar que saben se cazó en la región fue a principio de la década de 1980. La especie sobrevive en Talamanca al este de San Vito.

Puma concolor costarisensis Merriam 1901: El puma es una especie rara. El Dr. Luis Diego Gómez registró un ejemplar joven en el bosque del Jardín Botánico Wilson en 1999. Los informantes locales indicaron que se les encuentra en las laderas forestadas más inaccesibles de los ríos como el Coto Brus. Localidades: 1. Registros: 1.

\section{Familia Mephitidae}

Conepatus semistriatus trichurus Thomas 1902: El zorro hediondo fue una especie rara, que a pesar de intensos recorridos nocturnos sólo fue registrado una vez en un cafetal-bosque. Localidades: 3, 20. Ejemplares: 4.

Spilogale putorius celeris Hall 1938. El zorro hediondo manchado fue una especie rara, que a pesar de nuestros recorridos nocturnos sólo registramos en cafetales y cafetal-bosque. Localidades: 5,6, 17. Ejemplares: 4.

\section{Familia Mustelidae}

Eira barbara biologiae (Thomas 1900): A pesar de que el tolomuco fue raro en este estudio, es aparentemente común en la región, de acuerdo a los pobladores locales. Los registros incluyen a tres ejemplares que fueron observados en distintas ocasiones en cafetales muy perturbados, cercanos a remanentes de bosques riparios. En dos ocasiones se les observó en la mañana, a las 9 y 11 AM. Localidades: 3, 18. Ejemplares: 2. Hay registros previos en la región (Rodríguez y Chinchilla 1996, Wilson 1983).

Galictis vittata canaster Nelson 1901: El grisón es un animal aparentemente escaso en la región. En 1996 se observaron dos ejemplares en la mañana, en el Río Java en la EBC
(Hewson 1996). El Sr. Jorge Pérez indicó que son poco abundantes.

Lontra longicaudis annectens Major 1897: La nutria sureña es al parecer una especie común pero con densidades bajas en el área. La registramos en el bosque de la EBC y en bosques riparios. Se colectó un ejemplar que fue cazado porque había causado daños a un estanque en el que una familia mantenía carpas. En la región sobreviven desplazándose por ríos y arroyos que cruzan áreas deforestadas. Se encontraron rastros en el Río Java, en el Río Coto Brus y en varios arroyos de poca profundidad $(70 \mathrm{~cm})$ poca anchura $(1.20 \mathrm{~m})$. Localidades: 26, 33, 37, 38. Ejemplares: 4.

Mustela frenata costaricensis Goldman 1912: La comadreja fue el carnívoro más abundante, pero sólo se registró en cafetales y fragmentos de bosque con cafetales. Localidades: 3, 5, 6, 8, 17, 23, 25. Ejemplares: 8 .

\section{Familia Procyonidae}

Bassaricyon gabbii richardsoni J.A. Allen 1908: El olingo fue raro y solo se registró en el bosque de la EBC y un cafetal con fragmento de bosque. De acuerdo a los habitantes locales son relativamente comunes en la región. Localidades: 3, 4, 20. Ejemplares: 4.

Bassariscus sumichrasti notinus Thomas 1903. El cacomixtle es aparentemente raro en la región. No se registró en el trabajo de campo.

Nasua narica narica (Linnaeus 1776). El coatí o pizote es una especie común, que se registró en el bosque de la $\mathrm{EBC}$, fragmentos de bosque, cafetales y pastizales. Fue más común en el cafetal - bosque. En varias ocasiones los encontramos activos durante las primeras horas de la mañana. Localidades: 1, 3, 11, 17, 18, 20, 21, 22. Ejemplares: 16.

Potos flavus chiriquensis J.A. Allen 1904: La martilla fue común, con registros en el bosque de la EBC y en fragmentos de bosque. Es 
interesante que sobrevive en fragmentos pequeños, cercanos a casas, donde los pobladores locales no las molestan. En la región es simpátrica con Bassaricyon gabbii. Localidades: 3, 4, 18, 32, 41, 42, 43. Ejemplares: 13. Hay registros previos en la región (MNCR-428; Rodríguez y Chinchilla 1996, Wilson 1983).

Procyon lotor crassidens Hollister 1914. El mapache fue común en la región. Se le registro en el bosque de la EBC, fragmentos, cafetal y pastizal. Localidades: 4, 21, 27. Ejemplares: 3. Hay registros previos en la región (Rodríguez y Chinchilla 1996, Wilson 1983).

\section{ORDEN ARTIODACTYLA Familia Tayassuidae}

Tayassu pecari spiradens Goldman 1912: El jabalí de labios blancos fue exterminado desde la década de 1970, por la cacería indiscriminada y la destrucción del hábitat. Las poblaciones más cercanas confirmadas se localizan en la Península de la Osa.

Tayassu tajacu crusnigrum Bangs 1902: El pecarí de collar o saíno fue escaso. Hay registros recientes en la EBC y la finca de los Gamboa. No se le registro en el trabajo de campo.

\section{Familia Cervidae}

Mazama americana cerasina Hollister 1914. El cabrito de monte es una especie rara, a la que todavía se le caza en la región. Se registró de manera indirecta en el JBW y en el Río Cotos Brus. El guardabosques indicó que son escasos en la EBC. Todavía se les caza en la región. Localidades: 1, 37. Ejemplares: 2.

Odocoileus virginianus chiriquensis (J.A. Allen 1910) : El venado cola blanca es escaso. En el trabajo de campo sólo se registró por medio de un par de trofeos en una casa, que cazaron en la región, pero sin indicarnos la localidad precisa. Hay registros previos en la región (Rodríguez y Chinchilla 1996).

\section{ORDEN PERISSODACTILA Familia Tapiridae}

Tapirus bairdii (Gill 1865): El tapir esta localmente extinto. Los últimos ejemplares en la EBC fueron observados en la década de 1970. De acuerdo a nuestros informantes se les cazaba intensamente, especialmente en el Río Java y el Coto Brus.

\section{ORDEN RODENTIA \\ Familia Sciuridae}

Microsciurus alfari browni (Bangs 1902). La ardilla pigmea es aparentemente rara, pero esto tal vez se deba a sus hábitos, que hacen que sea difícil su observación. Sólo fue registrada dos veces, una en la EBC y otra un fragmento de bosque en Fila de Cal, en donde también se encontró a Sciurus granatensis, Cyclopes didactylus y Cebus capuccinus. Localidades: 1, 30. Ejemplares: 2.

Hay registros previos en la región en Agua Buena (Hall 1981) y San Vito (Wilson 1983). Registros adicionales: San Vito (LACM0287780).

Sciurus granatensis hoffmanni Peters 1863. La ardilla coliroja es abundante en la región. Esta especie se registró en numerosas ocasiones en la EBC, el JBW, fragmentos de bosque y cafetales. Es simpátrica con Microsciurus alfari. Localidades: 1, 2, 3, 4, 7, 19, 20, 21, 29, 32. Ejemplares: 43. Hay registros previos en la región (Rodríguez y Chinchilla 1996, Wilson 1983).

\section{Familia Heteromyidae}

Heteromys desmarestianus chiriquensis Enders 1938: A pesar de que se le encontró en este estudio, hay registros previos en la región (Wison 1983, Rodríguez y Chinchilla 1996). Es posible que su ausencia se deba a que no se muestreo en ambientes en los que es común. Hay registros previos en la región en Agua Buena (Hall 1981). 


\section{Familia Muridae}

Melanonys caliginosus chrisomelas J.A. Allen 1897. La rata morena fue abundante y se le registro en todos los tipos de vegetación con excepción del bosque continuo de la EBC. Fue simpátrica con Oligoryzomys fulvescens, Oryzomys alfaroi y Zygodontomys brevicauda. El peso promedio de 14 individuos fue de 42.6 g (intervalo 26 a $57 \mathrm{~g}$ ). Localidades: 6,13, 14, 20, 25, 26, 27. Ejemplares: 15. Registros adicionales: San Vito (KU-158324).

Nyctomys sumichrasti costariensis Goldman 1937: Esta especie fue rara, seguramente porque no se colectó en árboles. Los dos ejemplares se colectaron en un encino aproximadamente a $1.80 \mathrm{~cm}$ de altura y en una cabaña, respectivamente. Localidades: 1, 4. Registros: 2.

Oligoryzomys fulvescens costaricensis $\mathrm{J}$. A. Allen 1893. Este ratón fue una de las especies más abundantes, y se le registro en todos los tipos de vegetación.. Fue más abundante en el pastizal. Sus poblaciones aparentemente han aumentado como consecuencia de la conversión del bosque a pastizales y cultivos. El peso promedio de 76 individuos fue de $15.5 \mathrm{~g}$ (intervalo 10 a 35 g). Localidades: 11, 12, 13, 14, 17, 18, 19, 22, 23, 24, 25, 26, 27, 28. Ejemplares: 67. KU-160178.

Oryzomys couesi couesi (Alston 1877): Es una rata de campo común en ambientes perturbados. En el trabajo de campo se le registró en pastizales. Localidades: 3, 19, 28. Ejemplares: 5. Hay registros previos en la región (Rodríguez y Chinchilla 1996).

Oryzomys alfaroi alfaroi (J.A. Allen 1891): Esta rata de campo fue escasa y fue capturada en el bosque continuo y en ambientes perturbados. En abril del 2002 se encontraron dos nidos en un cultivo, bajo montones de vegetación. En uno de los nidos había 4 crías probablemente ya destetadas. Localidades: 3, 19, 28. Ejemplares: 5. Hay registros previos en la región en Agua Buena (Hall 1981).
Reithrodontomys mexicanus potrerograndei Goodwin 1945: La localidad tipo de esta especie es Agua Buena. No se le registró en el trabajo de campo, presumiblemente porque es una especie arborícola.

Scotinomys teguina apricus (Bangs 1902): Goodwin (1946) describió a una subespecie $(S$. t. endersi) con localidad típica a Agua Buena. Esta subespecie es sinónimo de $S$. $t$. apricus (Hall 1981). A pesar de los intensos muestreos en la región no se capturó ningún individuo. Es posible que sea ahora muy escasa por la extensa deforestación de la región. Hay registros previos en la región en Agua Buena (Hall 1981).

Sigmodon hispidus borucae J.A. Allen 1897: Esta rata fue registrada exclusivamente en pastizales. Es una especie que llega a ser muy abundante en sitios perturbados como pastizales y cultivos. Localidades: 14, 16. Ejemplares: 6.

Tylomys watsoni Thomas 1899. Esta rata arborícola fue rara; sólo se registraron dos individuos en la EBC y un fragmento de bosque en Fila de Cal. Sin embargo, es posible que sea más abundante, ya que no se muestreo intensamente el dosel, que es su hábitat principal. El ejemplar de la EBC se atrapó en una trampa colocada en un encino a dos metros de altura. El ejemplar de Fila de Cal, un juvenil, se colectó durante el día en un pedregal, completamente deforestado, a unos 60 metros del fragmento de bosque más cercano. Localidades: 3, 29. Ejemplares: 2.

Zygodontomys brevicauda cherrieri (J.A. Allen 1895): Esta rata fue común en ambientes perturbados, como pastizal y cafetal - bosque. Localidades: 8, 9, 14, 15, 21, 22. Ejemplares: 12 . Hay registros previos en la región (Rodríguez y Chinchilla 1996, Wilson 1983).

\section{Familia Erethizontidae}

Coendu mexicanus laenatus Thomas 1903: El puercoespín es bien conocido en la región. 
No se le registró en el trabajo de campo pero el Sr. Jorge Pérez, la Familia Gamboa y un guardabosques de la EBC indicaron que se le encuentra en la región pero que es escaso.

\section{Familia Dasyproctidae}

Dasyprocta puntata underwoodi Goldman 1931. La guatuza fue relativamente común en la $\mathrm{EBC}$, en fragmento de bosque, y en cafetales y pastizales adyacentes a bosques. En la EBC se le encontró frecuentemente en las primeras horas de la mañana. Localidades: 3, 4, 11, 20, 21, 22, 23. Ejemplares: 9.

\section{Familia Cuniculidae}

Cuniculus paca virgatus Bangs 1902. El tepezcuintle fue registrado en el bosque continuo, en fragmentos de bosque y en cafetales.
Es una especie apreciada por su carne, que todavía es cazada con perros. En un par de ocasiones se encontraron ejemplares en casas como mascota. Se usó el nombre de Cuniculus siguiendo la recomendación de la Comisión Internacional de Nomenclatura Zoológica (ICZN, 1998). Localidades: 2, 16, 18, 19, 21. Ejemplares: 8.

\section{ORDEN LAGOMORPHA Familia Leporidae}

Sylvilagus dicei: El conejo de monte fue una especie aparentemente común, especialmente en ambientes perturbados. En un par de ocasiones se les observó en la EBC durante el día, escondido entre la vegetación densa. Localidades: 4, 7, 9, 11, 13, 34. Ejemplares: 7. Hay registros previos en la región (Rodríguez y Chinchilla 1996, Wilson 1983). 


\section{APÉNDICE 4}

Localidades donde se registraron mamíferos en la región de San Vito de Coto Brus, Costa Rica.

\begin{tabular}{|c|c|c|c|c|c|}
\hline LOCALIDAD & ID & TIPO DE VEGETACIÓN & $\begin{array}{l}\text { LATITUD } \\
\text { (UTM) }\end{array}$ & $\begin{array}{l}\text { LONGITUD } \\
\text { (UTM) }\end{array}$ & $\begin{array}{l}\text { ALTITUD } \\
\text { (PIES) }\end{array}$ \\
\hline Estación Biológica Las Cruces & 1 & Bosque Premontano & $08^{\circ} 47^{\prime} 04^{\prime \prime}$ & $82^{\circ} 57^{\prime} 02^{\prime \prime}$ & 3400 \\
\hline Estación Biológica Las Cruces & 2 & Bosque Premontano & $08^{\circ} 47^{\prime} 06^{\prime \prime}$ & $82^{\circ} 58^{\prime} 07^{\prime \prime}$ & 3200 \\
\hline Estación Biológica Las Cruces & 3 & Bosque Premontano & $08^{\circ} 47^{\prime} 04^{\prime \prime}$ & $82^{\circ} 58^{\prime} 06^{\prime \prime}$ & 3100 \\
\hline Jardín Botánico Wilson & 4 & Bosque Premontano & $08^{\circ} 47^{\prime} 04^{\prime \prime}$ & $82^{\circ} 58^{\prime} 00^{\prime \prime}$ & 3100 \\
\hline Finca Gamboa, $2.6 \mathrm{~km}$ SW de San Vito & 5 & Cafetal & $08^{\circ} 47^{\prime} 08^{\prime \prime}$ & $82^{\circ} 58^{\prime} 44^{\prime \prime}$ & 3600 \\
\hline Fica Gamboa, $2.2 \mathrm{~km} \mathrm{~S}$ de San Vito & 6 & Cafetal & $08^{\circ} 48^{\prime} 09^{\prime \prime}$ & $82^{\circ} 58^{\prime} 32^{\prime \prime}$ & 3300 \\
\hline Linda Vista, $750 \mathrm{~m}$ SE de Las Cruces & 7 & Cafetal & $08^{\circ} 47^{\prime} 03^{\prime \prime}$ & $82^{\circ} 57^{\prime} 22^{\prime \prime}$ & 3400 \\
\hline $750 \mathrm{~m} \mathrm{~N}$ de Aguas Claras & 8 & Cafetal & $08^{\circ} 51^{\prime} 01^{\prime \prime}$ & $82^{\circ} 58^{\prime} 03^{\prime \prime}$ & 3800 \\
\hline 1. $3 \mathrm{~km} \mathrm{SE}$ de Aguas Claras & 10 & Cafetal & $08^{\circ} 50^{\prime} 04^{\prime \prime}$ & $82^{\circ} 57^{\prime} 41^{\prime \prime}$ & 2800 \\
\hline $1.2 \mathrm{~km} \mathrm{NE}$ de Aguas Claras & 11 & Cafetal & $08^{\circ} 50^{\prime} 03^{\prime \prime}$ & $82^{\circ} 57^{\prime} 54^{\prime \prime}$ & 2700 \\
\hline Melissa Meadow, $1.2 \mathrm{~km} \mathrm{SW}$ de Las Cruces & 12 & Pastizal inducido & $08^{\circ} 47^{\prime} 01^{\prime \prime}$ & $82^{\circ} 57^{\prime} 54^{\prime \prime}$ & 3600 \\
\hline Finca Gamboa, 2.2 km SE de San Vito & 13 & Pastizal inducido & $08^{\circ} 48^{\prime} 01^{\prime \prime}$ & $82^{\circ} 58^{\prime} 11^{\prime \prime}$ & 3400 \\
\hline Finca Gamboa, 2.1 km S de San Vito & 14 & Pastizal inducido & $08^{\circ} 48^{\prime} 02^{\prime \prime}$ & $82^{\circ} 58^{\prime} 29^{\prime \prime}$ & 3500 \\
\hline Finca Menonita, $550 \mathrm{~m}$ N de San Rafael & 15 & Pastizal inducido & $08^{\circ} 50^{\prime} 01^{\prime \prime}$ & $83^{\circ} 00^{\prime} 38^{\prime \prime}$ & 3400 \\
\hline 1.6 km NW de San Rafael & 16 & Pastizal inducido & $08^{\circ} 50^{\prime} 08^{\prime \prime}$ & $83^{\circ} 01^{\prime} 20^{\prime \prime}$ & 2600 \\
\hline 1.8 km NW de San Rafael & 17 & Pastizal inducido & $08^{\circ} 50^{\prime} 01^{\prime \prime}$ & $83^{\circ} 01^{\prime} 22^{\prime \prime}$ & 2700 \\
\hline Finca Gamboa, 2.5 km SE de San Vito & 18 & $\begin{array}{c}\text { Cafetal - fragmento } \\
\text { de bosque }\end{array}$ & $08^{\circ} 48^{\prime} 06^{\prime \prime}$ & $82^{\circ} 58^{\prime} 02^{\prime \prime}$ & 3500 \\
\hline Agua Buena, $650 \mathrm{~m}$ SW de Copal & 19 & $\begin{array}{c}\text { Cafetal - fragmento } \\
\text { de bosque }\end{array}$ & $08^{\circ} 46^{\prime} 03^{\prime \prime}$ & $82^{\circ} 57^{\prime} 19^{\prime \prime}$ & 3500 \\
\hline Linda Vista, $1.2 \mathrm{~km}$ E de Las Cruces & 20 & $\begin{array}{c}\text { Cafetal - fragmento } \\
\text { de bosque }\end{array}$ & $08^{\circ} 47^{\prime} 01^{\prime \prime}$ & $82^{\circ} 56^{\prime} 52^{\prime}$ & 3600 \\
\hline $750 \mathrm{~m} \mathrm{NE}$ de Aguas Claras & 21 & $\begin{array}{c}\text { Cafetal - fragmento } \\
\text { de bosque }\end{array}$ & $08^{\circ} 51^{\prime} 05^{\prime \prime}$ & $82^{\circ} 58^{\prime} 03^{\prime \prime}$ & 4070 \\
\hline $650 \mathrm{~m} \mathrm{NE}$ de Aguas Claras & 22 & $\begin{array}{l}\text { Cafetal - fragmento } \\
\text { de bosque }\end{array}$ & $08^{\circ} 51^{\prime} 07^{\prime \prime}$ & $82^{\circ} 58^{\prime} 09^{\prime \prime}$ & 2400 \\
\hline Manchuria, 1.2 km NE de San Rafael & 23 & $\begin{array}{c}\text { Cafetal - fragmento } \\
\text { de bosque }\end{array}$ & $08^{\circ} 50^{\prime} 05^{\prime \prime}$ & $83^{\circ} 00^{\prime} 46^{\prime \prime}$ & 3700 \\
\hline Finca Gamboa, $2 \mathrm{~km}$ SE de San Vito & 24 & $\begin{array}{l}\text { Pastizal inducido - } \\
\text { fragmento de bosque }\end{array}$ & $08^{\circ} 48^{\prime} 08^{\prime \prime}$ & $82^{\circ} 58^{\prime} 10^{\prime \prime}$ & 3400 \\
\hline $2.2 \mathrm{~km}$ SE de San Vito & 25 & $\begin{array}{l}\text { Pastizal inducido - } \\
\text { fragmento de bosque }\end{array}$ & $08^{\circ} 48^{\prime} 03^{\prime \prime}$ & $82^{\circ} 57^{\prime} 34^{\prime \prime}$ & 3300 \\
\hline Finca Gamboa, $1.7 \mathrm{~km} \mathrm{~S}$ de San Vito & 26 & $\begin{array}{l}\text { Pastizal inducido - } \\
\text { fragmento de bosque }\end{array}$ & $08^{\circ} 48^{\prime} 03^{\prime \prime}$ & $82^{\circ} 58^{\prime} 30^{\prime \prime}$ & 3100 \\
\hline Manchuria, 4.2 km SE de la Unión & 27 & $\begin{array}{l}\text { Pastizal inducido - } \\
\text { fragmento de bosque }\end{array}$ & $08^{\circ} 50^{\prime} 00^{\prime \prime}$ & $83^{\circ} 00^{\prime} 40^{\prime \prime}$ & 4200 \\
\hline
\end{tabular}




\section{APÉNDICE 4 (continuación)}

\section{LOCALIDAD}

Manchuria, 4.4 km SE de la Unión

Manchuria 4.7 km SE de La Unión

Cuesta Fila de Cal, 1.2 km al N de Caño Seco

Agua Buena, 650 m SW de Copal

Quebrada Pavo, 1 km NE de San Vito

Rio Sabalito, 300 m E San Joaquín

Copal

Río Limón, 3 km SE de San Luis

Río Coto Brus, Bonanza

Río Coto Brus, $3.5 \mathrm{~km}$ al E de Jabillo

Río Java, EBC

1 km NW de Santa Cecilia

Santo Domingo

Agua Buena, 650 m SW de Copal

$1.2 \mathrm{~km}$ E de Los Angeles

$1.6 \mathrm{~km} \mathrm{NE}$ de Sabalito

Agua Buena, 150 m S de Linda Vista

\section{ID TIPO DE VEGETACIÓN}

Pastizal inducido fragmento de bosque

Pastizal inducido fragmento de bosque

Fragmento de bosque Acahual

Fragmento de bosque

Fragmento de bosque

Fragmento de bosque

Fragmento de bosque

Fragmento de bosque

Fragmento de bosque

Fragmento de bosque

Fragmento de bosque

Fragmento de bosque

Fragmento de bosque

Fragmento de bosque

Fragmento de bosque

Cafetal

\section{LATITUD LONGITUD ALTITUD \\ (UTM) \\ (UTM) \\ (PIES)}

08 $500^{\prime} 01^{\prime \prime} \quad 83^{\circ} 00^{\prime} 41^{\prime \prime}$

2400

0850'59" $\quad 83^{\circ} 00^{\prime} 48^{\prime \prime}$

3000

084'47" 8257'01"

$08^{\circ} 46^{\prime} 35^{\prime \prime} \quad 82^{\circ} 57^{\prime} 24^{\prime \prime}$

084' $47^{\prime \prime} \quad 82^{\circ} 58^{\prime} 08^{\prime \prime}$

084' $35^{\prime \prime} \quad 82^{\circ} 57^{\prime} 13^{\prime \prime}$

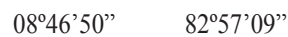

08 51'02" 8305'02"

08 $56^{\circ} 47^{\prime \prime} \quad 83^{\circ} 02^{\prime} 49^{\prime \prime}$

085' $13^{\circ} " \quad 83^{\circ} 03^{\prime} 40^{\prime \prime}$

08ㄴㄱ' $82^{\circ} 58^{\prime} 10^{\prime \prime}$

08'53'34" 8304'34"

084ㄴ $42^{\prime \prime} \quad 82^{\circ} 58^{\prime} 26^{\prime \prime}$

4200

08ㄴㅇ' $38^{\prime \prime} \quad 82^{\circ} 57^{\prime} 26^{\prime \prime}$

$08^{\circ} 47^{\prime} 20^{\prime \prime} \quad 82^{\circ} 56^{\prime} 07^{\prime \prime}$

3300

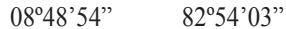

$08^{\circ} 48^{\prime} 27^{\prime \prime} \quad 82^{\circ} 57^{\prime} 38^{\prime \prime}$

3600 